\title{
NAMPT/Visfatin expression by inflammatory monocytes mediates arthritis pathogenesis by promoting IL-17-producing T cells
}

\author{
Jessy Présumey ${ }^{1,2}$, Gabriel Courties ${ }^{1,2}$, Pascale Louis-Plence ${ }^{1,2}$, Virginie Escriou ${ }^{3,4,5,6}$, Daniel Scherman ${ }^{3,4,5,6}$, \\ Yves-Marie Pers ${ }^{1,2,7}$, Hans Yssel ${ }^{1,2}$, Jérôme Pène ${ }^{1,2}$, Diego Kyburz ${ }^{8}$, Steffen Gay ${ }^{8}$, Christian Jorgensen 1,2,7, \\ Florence Apparailly ${ }^{1,2,7^{*}}$
}

From 7th European Workshop on Immune-Mediated Inflammatory Diseases

Noordwijk aan Zee, the Netherlands. 28-30 November 2012

\section{Background}

Nicotinamide phosphoribosyltransferase (NAMPT)/PBEF/ Visfatin exerts multiple functions and has been implicated in the pathogenesis of rheumatoid arthritis. The expression of NAMPT is increased during inflammation and is identified as a novel mediator of innate immunity. To gain insight into its role in arthritis and given that NAMPT induces IL-6 expression that is critical for Th17 lymphocytes, we hypothesized that NAMPT-stimulated production of IL- 6 by monocytes might in turn promote Th17 cells.

\section{Materials and methods}

siRNA uptake and NAMPT expression were determined in Ly6 $\mathrm{C}^{\text {high }}$ and Ly6 $\mathrm{C}^{\text {low }}$ monocyte subsets following intravenous injection of siRNA against NAMPT (siNAMPT) or non-targeting siRNA (siCT) formulated with the DMAPAP cationic liposome into mice. Mice with established collagen-induced arthritis (CIA) were treated weekly after disease onset with siNAMPT or siCT and clinical features were assessed. $\mathrm{T}$ helper cell frequencies, cytokine production and percentage of IL-6-producing Ly6 $\mathrm{C}^{\text {high }}$ monocytes were analyzed. Using a coculture system consisting of purified $\mathrm{CD} 14^{+}$monocytes and autologous $\mathrm{CD} 4^{+}$ $\mathrm{T}$ cells, NAMPT and cytokine production, as well as the percentage of IL-17-producing $\mathrm{CD} 4^{+} \mathrm{T}$ cells were determined following transfection of $\mathrm{CD} 14^{+}$monocytes with siCT or siNAMPT.

\section{Results}

Upon intravenous injection, siRNA was preferentially engulfed by Ly $6 C^{\text {high }}$ monocytes and siRNA-mediated silencing of NAMPT expression in Ly6 $\mathrm{C}^{\text {high }}$ monocytes reduced IL- 6 production by these cells, mitigated Th17 cell expansion, and inhibited inflammatory features and CIA progression. Moreover, NAMPT-RNAi-silenced $\mathrm{CD} 14^{+}$monocytes were found to reduce the percentage of IL-17-producing $\mathrm{CD}^{+}{ }^{+} \mathrm{T}$ cells.

\section{Conclusions}

Taken together, our results show that the expression of NAMPT in Ly6C ${ }^{\text {high }}$ monocytes promotes Th17 cells. Our findings provide new mechanistic insight into the action of NAMPT in arthritis and demonstrate the utility of targeting disease-causing genes in Ly6C $\mathrm{C}^{\text {high }}$ monocytes for therapeutic intervention in arthritis.

\section{Author details}

${ }^{1}$ INSERM, U844, Montpellier, France. ${ }^{2}$ University of Medicine, Montpellier, France. ${ }^{3}$ INSERM, U1022, UFR des Sciences Pharmaceutiques et Biologiques, Paris, France. ${ }^{4}$ CNRS, UMR8151, Paris, France. ${ }^{5}$ University of Pharmacy Paris Descartes, Paris, France. ${ }^{6}$ Ecole Nationale Supérieure de Chimie de Paris, Paris, France. ${ }^{7}$ University Hospital of Montpellier, Clinical Dept. for osteoarticular diseases, Montpellier, France. ${ }^{8}$ Center of Experimental Rheumatology, University Hospital Zurich and Zurich Center of Integrative Human Physiology, Zurich, Switzerland.

Published: 28 November 2012

doi:10.1186/1479-5876-10-S3-P48

Cite this article as: Présumey et al.: NAMPT/Visfatin expression by inflammatory monocytes mediates arthritis pathogenesis by promoting IL-17-producing T cells. Journal of Translational Medicine 2012 10(Suppl 3): P48. 